\title{
Rosai-Dorfman Disease Presenting with Solitary Liver Mass Without Lymphadenopathy: Case Report and Literature Review
}

\author{
Yousef Roosta ${ }^{1}$, Ali Esfahani², Amir Vahedi ${ }^{3}$, Kosar Tarvirizadeh ${ }^{2}$, Sadegh Asoubar ${ }^{2}$, \\ Behdad Boroofeh $^{1}$, Roshan Dinparast ${ }^{1}$, Farhad Behzadi ${ }^{1}$, Mortaza Raeisi ${ }^{2}$, Mohammadreza \\ Mohammad Hosseiniazar ${ }^{1}$, and Amin Sedokani ${ }^{1}$ \\ ${ }^{1}$ Urmia University of Medical Sciences \\ ${ }^{2}$ Tabriz University of Medical Sciences \\ ${ }^{3}$ Tabriz Medical University
}

February 6, 2021

\begin{abstract}
This care report presents a 51-year-old woman patient with a cyst-like mass in the liver. The proliferated histiocytes, with the eosinophilic nucleolus, occasionally showing lymphophagocytosis (emperipolesis) positively for CD68 protein S-100 were reported in the pathological study, and the plasma cells were positive for CD138, consistent with Rosai-Dorfman disease.

Rosai-Dorfman Disease Presenting with Solitary Liver Mass Without Lymphadenopathy: Case Report and Literature Review

Yousef Roosta $^{1}$, Ali Esfahani ${ }^{2}$, Amir Vahedi ${ }^{3}$, Kosar Tarvirizadeh ${ }^{4}$, Sadegh Asoubar ${ }^{5}$, Behdad Boroofeh ${ }^{6}$, Roshan Dinparast ${ }^{7}$, Farhad Behzadi ${ }^{8}$, Mortaza Raeisi ${ }^{9}$, Mohammadreza Mohammad Hosseiniazar ${ }^{10}$, Amin Sedokani ${ }^{11}$

1 Internal Medicine Department, Urmia University of Medical Sciences, Urmia, Iran. Email:yroosta@gmail.com

${ }^{2}$ Hematology and Oncology Research Center, Tabriz University of Medical Sciences, Tabriz, Iran. Email:ali_sfhn@yahoo.com

3 Department of Clinical Pathology, Tabriz University of Medical Sciences, Tabriz, Iran. Email:amirvahedy@gmail.com

4 Medicine Faculty, Tabriz University of Medical Science, Tabriz, Iran. Email:kawsar.tarvirdizade@yahoo.com

5 Hematology and Oncology Research Center, Tabriz University of Medical Sciences, Tabriz, Iran. Email:asoubarsadegh@gmail.com

6 Internal Medicine Department, Urmia University of Medical Sciences, Urmia, Iran. Email:b.boroofeh@yahoo.com

7 Internal Medicine Department, Urmia University of Medical Sciences, Urmia, Iran. Email:roshan.dinparst@gmail.com

8 Internal Medicine Department, Urmia University of Medical Sciences, Urmia, Iran. Email:Farhad.behzadi.md@gmail.com
\end{abstract}


9 Hematology and oncology research center, Tabriz university of medical sciences, Tabriz, IRAN, Email:Raiisy@yahoo.com

10 Internal Medicine Department, Urmia University of Medical Sciences, Urmia, Iran. Email:mohammadazar@gmail.com

11 Cardiology Department, Urmia University of Medical Sciences, Urmia, IRAN.Email:A.sedokani@gmail.com

*Corresponding Author: Amin Sedokani

Medical Doctor, Cardiology Department, Urmia University of Medical Sciences, Urmia, IRAN.

Email: A.sedokani@gmail.com

Address: 17 Sharivar St., Urmia, IRAN (Postal Code: 571478334)

Phone: +984432375907

Fax: +984432372917

\section{Abstract}

This care report presents a 51-year-old woman patient with a cyst-like mass in thew liver. The proliferated histiocytes, with the eosinophilic nucleolus, occasionally showing lymphophagocytosis (emperipolesis) positively for CD68 protein S-100 were reported in the pathological study, and the plasma cells were positive for CD138, consistent with Rosai-Dorfman disease.

\section{Keywords}

Rosai-Dorfman Disease, Destombes-Rosai-Dorfman Disease, Histiocytosis, Liver Mass

\section{Introduction}

Rosai-Dorfman disease, or sinus histiocytosis with massive lymphadenopathy, is characterized by histiocytosis in different organs specially in lymph nodes ${ }^{1,2}$. The exact etiology of the disease is unknown but most of the patients present with fever, leukocytosis, and nonpainful cervical lymphadenopathy. It can involve the skin, soft tissue or less commonly central nervous system. The gastrointestinal engagement is rare ${ }^{3-7}$. This article is a rare case report of solitary liver mass without lymphadenopathy with a review of previous literature.

\section{Case Presentation}

A 51-year-old lady was admitted to hospital with chronic abdominal pain, weight loss (5\% of body weight), history of undulant fever and nocturnal sweating. In physical examination, orthostatic hypotension, conjunctival pallor and abdominal right upper quadrant tenderness were noticed. Laboratory tests showed elevated Erythroid Sedimentation Rate $\left(\mathrm{ESR}=87^{\mathrm{mm}} / \mathrm{h}\right)$ and normocytic normochromic anemia (hemoglobin=9.3 $\mathrm{g} / \mathrm{dl}$ ). Abdominal ultrasound revealed a $37 \mathrm{~mm} \times 49 \mathrm{~mm}$ cyst-like mass in the left lobe of liver (4A segment) without internal septation, and no other abnormality in abdominopelvic cavity was identified. Computed tomography showed a non-enhanced hypodense mass with regular margin (sized: $\left.4 \times 5^{\mathrm{cm}}\right)$ in the left lobe of liver (Figure 1) without any further abnormal findings. 
We re-reviewed patient's past medical history for any diagnostic clues for the solitary liver mass. Personal and family history was negative for chronic liver disease and viral hepatitis risk factors. No stigmata for cirrhosis were identified. Viral hepatitis panel, alpha fetoprotein and CA19-9 were undetectable. Screening colonoscopy for colorectal cancer was normal 6 months before symptoms start.

Finally, she was admitted to Ghazi Hospital, affiliated to Tabriz University of Medical Sciences, for diagnostic mass resection and underwent partial hepatectomy surgery. In pathological study, the liver tissue contained a cystic lesion composed of proliferated histiocytes, with eosinophilic nucleolus, some double or multinucleated, occasionally showing lymphophagocytosis (emperipolesis). The histiocytes were mixed with a polymorph infiltration composed of mononuclear and plasma cells. Histiocytes stained positively for CD68 and protein S-100, and plasma cells were positive for CD138, kappa and lambda. Hodgkin's lymphoma was ruled out by negative CD15 and CD30 staining. Peripheral blood smear and bone marrow aspiration were normal. Bone marrow biopsy showed lymphoplasmacytosis with slight megakaryocytosis. The liver histological pattern was consistent with Rosai-Dorfman disease (RDD). The patient was consequently referred to the hematology service and we decided to start prednisone, $30^{\mathrm{mg} / \text { day }}$. She was followed conservatively with repeat imaging after 3 months. The follow-up CT revealed no recurrence and she responded clinically to steroids. She continues to be followed with yearly imaging and $5^{\mathrm{mg} / \text { day }}$ prednisone and will likely remain on steroids for the long term.

\section{Discussion}

Rosai-Dorfman Disease (RDD) which is also referred to as sinus histiocytosis with massive lymphadenopathy, is an exceedingly rare non-Langerhans cell reactive histiocytic disorder that was initially described in $1969^{2,8}$. The prevalence of RDD is approximately 1:200,000 in the United States, and may present with fever, neutrophilia, increased serum erythrocyte sedimentation rate, leukocytosis, lymphopenia, polyclonal hyperglobulinemia, and anemia. In most cases, however, there is no apparent or specific symptom ${ }^{9-13}$.

In its most recent revised classification in 2016, the writing group of the Histiocyte Society has classified RDD into the following subtypes: familial RDD, classical RDD, extranodal RDD, neoplasia-associated RDD, and immune disease-associated RDD ${ }^{14}$. Classic RDD presents with massive painless cervical lymphadenopathy in children and young adults, with a slight (58\%) male predominance and a benign self-limiting course $8,13,15,16$. Older age and underlying immunologic abnormalities (autoimmune hemolytic anemia, WiskottAldrich syndrome, glomerulonephritis, rheumatoid arthritis) are associated with a more extensive disease and chronic relapsing course ${ }^{17}$.

The pathologic features of nodal RDD include the sinus expansion of large histiocytes, described by Destombes as possessing "watery-clear" cytoplasm with a large foamy nucleus and prominent nucleolus. Consistent features, regardless of the site, include the cytomorphology of the large pale histiocytes and their immunophenotype. Emperipolesis, the trafficking of intact leukocytes through the cytoplasm, is a helpful finding but is not required for diagnosis, because it can be focal, especially at extranodal sites, and may be seen focally in other histiocytoses such as juvenile xanthogranuloma, and malignant histiocytoses. Extranodal lesions are usually associated with more fibrosis, fewer RDD histiocytes and less emperipolesis. In such cases, immunostains are needed to highlight the residual RDD histiocytes in a rich lymphoplasmacytic background with stromal fibrosis and a variable xanthomatous histiocytic reaction. The immunophenotype of the large RDD histiocytes is characterized by cytoplasmic and nuclear S100 and fascin positivity, with CD68 and variable CD163 and CD14 positivity. The cells are CD1a-/CD207- in contrast to Langerhans cell histiocytosis ${ }^{18-22}$.

Extranodal involvement is common and may occur in more than $40 \%$ of patients, sometimes without associated lymphadenopathy, and only $23 \%$ of patients have exclusive extranodal disease ${ }^{13,23,24}$. Virtually every organ can be affected by RDD (respiratory, bone, CNS, genitourinary, orbit, soft tissue, visceral organs, nasal cavity, etc.) and in contrast to isolated lymphadenopathy, the mortality of extranodal RDD is higher in patients with lower respiratory tract, hepatic, or renal involvement $(30 \%, 33 \% \text {, and } 40 \% \text {, respectively })^{13}$. 
Intraabdominal extranodal disease is uncommon, with an incidence of $4 \%^{25}$. The gastrointestinal (GI) system is one of the least commonly affected sites $(<1 \%)$; we are aware of only 41 examples reported in the English literature $7,13,25-40$. The GI disease mostly affects middle-aged females. Most digestive system cases arise in the tubular GI tract with most cases being located beyond the pylorus ${ }^{40}$. Pancreatic or hepatic involvement is reported but extremely rare ${ }^{13,34,41}$. Hepatic lesions tend to present in younger patients; all but the 1 reported patient has had systemic disease ${ }^{25,29,33}$. To our knowledge, our case is the first adult patient reported with solitary liver RDD, without lymphadenopathy.

No uniform approach has been delineated for RDD, and treatment is best tailored to the individual clinical circumstances. Therapeutic strategies in literature include: observation, steroids (prednisone $40-70^{\mathrm{mg}}$ per day, followed by taper), surgical resection/debulking, radiotherapy, chemotherapy, Sirolimus, Thalidomide, Rituximab and Imatinib ${ }^{42-49}$. In our case, the patient had a partial response to steroids and her symptoms have been improving. The authors do not expect a complete resolution of the disease, however in cases such as these we recommend beginning with steroids and escalating to other treatments only if the disease is non-responsive to steroids and difficult to manage or life threatening. We encourage serial imaging and follow-up for any changes.

\section{Declarations}

\section{Ethics approval and consent to participate}

The patient's identity is secret and preserved unknown in the article and the patient received an oral and written permission form that was approved by the ethics committee of Tabriz University of medical sciences and Urmia university of medical sciences. The consent was obtained from the study participant prior to study commencement and the study participants gave consent to publish.

\section{Consent to publish}

Signed consent for publication have been obtained from the patient and legal guardian.

\section{Availability of data and material}

Data sharing not applicable to this article as no datasets were generated or analyzed during the current study.

\section{Competing interests}

All of authors report no kind of conflict of interests in this study.

\section{Funding}

This study was funded by Urmia University of Medical Sciences and there is no other organizational or governmental funding.

\section{Authors' Contributions}

All of the authors in this study have contributed equally in design, performance, data collection, and writing and review of the manuscript. 


\section{Acknowledgements}

We would like to thank the health care personnel of Tabriz and Urmia Universities of Medical Sciences.

\section{References}

1. Dalia S, Sagatys E, Sokol L, Kubal T. Rosai-Dorfman disease: tumor biology, clinical features, pathology, and treatment. Cancer Control . Oct 2014;21(4):322-7. doi:10.1177/107327481402100408

2. Destombes P. [Adenitis with lipid excess, in children or young adults, seen in the Antilles and in Mali. (4 cases)]. Bull Soc Pathol Exot Filiales. Nov-Dec 1965;58(6):1169-75. Adenites avec surcharge lipidique, de l'enfant ou de l'adulte jeune, observees aux Antilles et au Mali. (Quatre observations).

3. Cooper SL, Jenrette JM. Rosai-Dorfman disease: management of CNS and systemic involvement. Clin Adv Hematol Oncol . Mar 2012;10(3):199-202.

4. Frater JL, Maddox JS, Obadiah JM, Hurley MY. Cutaneous Rosai-Dorfman disease: comprehensive review of cases reported in the medical literature since 1990 and presentation of an illustrative case. $J$ Cutan Med Surg . Nov-Dec 2006;10(6):281-90. doi:10.2310/7750.2006.00067

5. McClellan SF, Ainbinder DJ. Orbital Rosai-Dorfman disease: a literature review. Orbit . Oct 2013;32(5):341-6. doi:10.3109/01676830.2013.814689

6. Sandoval-Sus JD, Sandoval-Leon AC, Chapman JR, et al. Rosai-Dorfman disease of the central nervous system: report of 6 cases and review of the literature. Medicine (Baltimore). May 2014;93(3):165-75. doi:10.1097/MD.0000000000000030

7. Zhao M, Li C, Zheng J, et al. Extranodal Rosai-Dorfman disease involving appendix and mesenteric nodes with a protracted course: report of a rare case lacking relationship to IgG4-related disease and review of the literature. International journal of clinical and experimental pathology . 2013;6(11):2569-77.

8. Rosai J, Dorfman RF. Sinus histiocytosis with massive lymphadenopathy. A newly recognized benign clinicopathological entity. Archives of pathology . Jan 1969;87(1):63-70.

9. Abla O, Jacobsen E, Picarsic J, et al. Consensus recommendations for the diagnosis and clinical management of Rosai-Dorfman-Destombes disease. Blood . Jun 28 2018;131(26):2877-2890. doi:10.1182/blood2018-03-839753

10. Xu H, Zhang F, Lu F, Jiang J. Spinal Rosai-Dorfman disease: case report and literature review. European spine journal : official publication of the European Spine Society, the European Spinal Deformity Society, and the European Section of the Cervical Spine Research Society . May 2017;26(Suppl 1):117-127. doi:10.1007/s00586-017-4975-0

11. Igrutinovic Z, Medovic R, Markovic S, et al. Rosai-Dorfman disease of vertebra: Case report and literature review. The Turkish journal of pediatrics . 2016;58(5):566-571. doi:10.24953/turkjped.2016.05.020

12. Kozak B, Talbott J, Uzelac A, Rehani B. Rosai-Dorfman Disease Isolated to the Thoracic Epidural Spine. Journal of radiology case reports . Nov 2015;9(11):6-16. doi:10.3941/jrcr.v9i11.2629

13. Foucar E, Rosai J, Dorfman R. Sinus histiocytosis with massive lymphadenopathy (Rosai-Dorfman disease): review of the entity.Seminars in diagnostic pathology . Feb 1990;7(1):19-73.

14. Emile JF, Abla O, Fraitag S, et al. Revised classification of histiocytoses and neoplasms of the macrophage-dendritic cell lineages.Blood . Jun 2 2016;127(22):2672-81. doi:10.1182/blood-2016-01-690636

15. McAlister WH, Herman T, Dehner LP. Sinus histiocytosis with massive lymphadenopathy (RosaiDorfman disease). Pediatric radiology . 1990;20(6):425-32. doi:10.1007/bf02075199 
16. Sodhi KS, Suri S, Nijhawan R, Kang M, Gautam V. Rosai-Dorfman disease: unusual cause of diffuse and massive retroperitoneal lymphadenopathy. The British journal of radiology . Sep 2005;78(933):845-7. doi:10.1259/bjr/23127241

17. Mar WA, Yu JH, Knuttinen MG, et al. Rosai-Dorfman Disease: Manifestations Outside of the Head and Neck. AJR American journal of roentgenology . Apr 2017;208(4):721-732. doi:10.2214/ajr.15.15504

18. Remadi S, Anagnostopoulou ID, Jlidi R, Cox JN, Seemayer TA. Extranodal Rosai-Dorfman disease in childhood. Pathology, research and practice. Oct 1996;192(10):1007-15. doi:10.1016/s0344-0338(96)80042-2

19. Paulli M, Locatelli F, Kindl S, et al. Sinus histiocytosis with massive lymphoadenopathy (RosaiDorfman disease). Clinico-pathological analysis of a paediatric case. European journal of pediatrics . Sep 1992;151(9):672-5. doi:10.1007/bf01957571

20. Foss HD, Herbst H, Araujo I, et al. Monokine expression in Langerhans' cell histiocytosis and sinus histiocytosis with massive lymphadenopathy (Rosai-Dorfman disease). The Journal of pathology . May 1996;179(1):60-5. doi:10.1002/(sici)1096-9896(199605)179:1<60::Aid-path533>3.0.Co;2-f

21. Wang KH, Cheng CJ, Hu CH, Lee WR. Coexistence of localized Langerhans cell histiocytosis and cutaneous Rosai-Dorfman disease.The British journal of dermatology . Oct 2002;147(4):770-4. doi:10.1046/j.1365-2133.2002.04879.x

22. Wenig BM, Abbondanzo SL, Childers EL, Kapadia SB, Heffner DR. Extranodal sinus histiocytosis with massive lymphadenopathy (Rosai-Dorfman disease) of the head and neck. Human pathology . May 1993;24(5):483-92. doi:10.1016/0046-8177(93)90160-i

23. Rodriguez-Galindo C, Helton KJ, Sanchez ND, Rieman M, Jeng M, Wang W. Extranodal Rosai-Dorfman disease in children. Journal of pediatric hematology/oncology . Jan 2004;26(1):19-24. doi:10.1097/00043426200401000-00007

24. Gaitonde S. Multifocal, extranodal sinus histiocytosis with massive lymphadenopathy: an overview. Archives of pathology \& laboratory medicine. Jul 2007;131(7):1117-21. doi:10.1043/15432165(2007)131[1117:Meshwm]2.0.Co;2

25. Lauwers GY, Perez-Atayde A, Dorfman RF, Rosai J. The digestive system manifestations of RosaiDorfman disease (sinus histiocytosis with massive lymphadenopathy): review of 11 cases. Human pathology . Mar 2000;31(3):380-5. doi:10.1016/s0046-8177(00)80254-3

26. Romero Arenas MA, Singhi AD, Hruban RH, Cameron AM. Rosai-dorfman disease (sinus histiocytosis with massive lymphadenopathy) of the pancreas: third reported occurrence. Journal of gastrointestinal cancer. Dec 2012;43(4):626-9. doi:10.1007/s12029-012-9424-z

27. Alatassi H, Ray MB, Galandiuk S, Sahoo S. Rosai-Dorfman disease of the gastrointestinal tract: report of a case and review of the literature. International journal of surgical pathology. Jan 2006;14(1):95-9. doi:10.1177/106689690601400119

28. Baran B, Karaca C, Soyer OM, et al. Rosai-Dorfman disease with diffuse gastrointestinal involvement. European journal of gastroenterology 85 hepatology . Jul 2013;25(7):869-74. doi:10.1097/MEG.0b013e32836019f8

29. Di Tommaso L, Rahal D, Bossi P, Roncalli M. Hepatic rosai-dorfman disease with coincidental lymphoma: report of a case.International journal of surgical pathology . Dec 2010;18(6):540-3. doi:10.1177/1066896908329590

30. Esquivel J, Krishnan J, Jundi M, Sugarbaker PH. Rosai-Dorfman disease (sinus histiocytosis with massive lymphadenopathy) of the pancreas: first case report. Hepato-gastroenterology . Mar-Apr 1999;46(26):1202-5.

31. Ide M, Asao T, Yoshida T, et al. Rosai-Dorfman disease of the colon presented as small solitary polypoid lesion. Rare tumors . Mar 31 2010;2(1):e2. doi:10.4081/rt.2010.e2 
32. Long E, Lassalle S, Cheikh-Rouhou R, Hofman V, Lacour JP, Hofman P. Intestinal occlusion caused by Rosai-Dorfman disease mimicking colonic diverticulitis. Pathology, research and practice . 2007;203(4):233-7. doi:10.1016/j.prp.2007.01.008

33. Maheshwari A, Seth A, Choudhury M, et al. Rosai-Dorfman disease: a case with lymphadenopathy and liver involvement. Journal of pediatric hematology/oncology . Mar 2009;31(3):200-2. doi:10.1097/MPH.0b013e31818e5369

34. Mantilla JG, Goldberg-Stein S, Wang Y. Extranodal Rosai-Dorfman Disease: Clinicopathologic Series of 10 Patients With Radiologic Correlation and Review of the Literature. American journal of clinical pathology . Feb 2016;145(2):211-21. doi:10.1093/ajcp/aqv029

35. Meindl A, Rao MS, Yang GY. Extranodal Rosai-Dorfman Disease With Mucosal Involvement of the Stomach in a Background of Autoimmune Atrophic Gastritis. International journal of surgical pathology . Oct 2018;26(7):671-675. doi:10.1177/1066896918773399

36. Podberezin M, Angeles R, Guzman G, Peace D, Gaitonde S. Primary pancreatic sinus histiocytosis with massive lymphadenopathy (Rosai-Dorfman disease): an unusual extranodal manifestation clinically simulating malignancy. Archives of pathology $\& 3$ laboratory medicine. Feb 2010;134(2):276-8. doi:10.1043/1543$2165-134.2 .276$

37. Shukla D, Veillon DM, Abreo F, Cotelingam JD. Pathologic quiz case: a 55-year-old woman with a history of treated Hodgkin disease and a persistent abdominal mass. Extranodal gastrointestinal RosaiDorfman disease. Archives of pathology \& laboratory medicine . Nov 2003;127(11):1527-8. doi:10.1043/15432165(2003)127<1527:Pqcayw >2.0.Co;2

38. Wimmer DB, Ro JY, Lewis A, et al. Extranodal rosai-dorfman disease associated with increased numbers of immunoglobulin g4 plasma cells involving the colon: case report with literature review. Archives of pathology \& laboratory medicine. Jul 2013;137(7):999-1004. doi:10.5858/arpa.2011-0547-CR

39. Zivin SP, Atieh M, Mosier M, Paner GP, Aranha GV. Rosai-Dorfman disease (sinus histiocytosis with massive lymphadenopathy) of the pancreas: second case report. Journal of gastrointestinal surgery : official journal of the Society for Surgery of the Alimentary Tract . Apr 2009;13(4):806-9. doi:10.1007/s11605-008$0752-\mathrm{z}$

40. Alruwaii ZI, Zhang Y, Larman T, Miller JA, Montgomery EA. Rosai-Dorfman Disease of the Digestive System-Beware Vasculopathy: A Clinicopathologic Analysis. The American journal of surgical pathology . Dec 2019;43(12):1644-1652. doi:10.1097/pas.0000000000001343

41. Shaikh F, Awan O, Mohiuddin S, Farooqui S, Khan SA, McCartney W. 18F-FDG PET/CT Imaging of Extranodal Rosai-Dorfman Disease with Hepatopancreatic Involvement - A Pictorial and Literature Review.Cureus . Dec 3 2015;7(12):e392. doi:10.7759/cureus.392

42. Pulsoni A, Anghel G, Falcucci P, et al. Treatment of sinus histiocytosis with massive lymphadenopathy (Rosai-Dorfman disease): report of a case and literature review. American journal of hematology . Jan 2002;69(1):67-71. doi:10.1002/ajh.10008

43. Forest F, N'Guyen A T, Fesselet J, et al. Meningeal Rosai-Dorfman disease mimicking meningioma. Annals of hematology . Jun 2014;93(6):937-40. doi:10.1007/s00277-013-1994-8

44. Z'Graggen WJ, Sturzenegger M, Mariani L, Keserue B, Kappeler A, Vajtai I. Isolated RosaiDorfman disease of intracranial meninges.Pathology, research and practice . 2006;202(3):165-70. doi:10.1016/j.prp.2005.11.004

45. Al-Khateeb TH. Cutaneous Rosai-Dorfman Disease of the Face: A Comprehensive Literature Review and Case Report. Journal of oral and maxillofacial surgery : official journal of the American Association of Oral and Maxillofacial Surgeons . Mar 2016;74(3):528-40. doi:10.1016/j.joms.2015.09.017 
46. Chen HH, Zhou SH, Wang SQ, Teng XD, Fan J. Factors associated with recurrence and therapeutic strategies for sinonasal Rosai-Dorfman disease. Head $\&$ neck . Oct 2012;34(10):1504-13. doi:10.1002/hed.21832

47. Maklad AM, Bayoumi Y, Tunio M, Alshakweer W, Dahar MA, Akbar SA. Steroid-resistant extranodal rosai-dorfman disease of cheek mass and ptosis treated with radiation therapy. Case reports in hematology . 2013;2013:428297. doi:10.1155/2013/428297

48. Horneff G, Jurgens H, Hort W, Karitzky D, Gobel U. Sinus histiocytosis with massive lymphadenopathy (Rosai-Dorfman disease): response to methotrexate and mercaptopurine. Medical and pediatric oncology . Sep 1996;27(3):187-92. doi:10.1002/(sici)1096-911x(199609)27:3<187::Aid-mpo10>3.0.Co;2-d

49. Tasso M, Esquembre C, Blanco E, Moscardo C, Niveiro M, Paya A. Sinus histiocytosis with massive lymphadenopathy (Rosai-Dorfman disease) treated with 2-chlorodeoxyadenosine. Pediatric blood \& cancer . Oct 15 2006;47(5):612-5. doi:10.1002/pbc.20668

Figure Legends

Figure 1: Computed tomography showed a non-enhanced hypodense mass.

Figure2: Histiocytes, with the eosinophilic nucleolus, some double or multinucleated, occasionally showing lymphophagocytosis (emperipolesis).

Figure3: Histiocytes stained positively for CD68 and protein S-100.

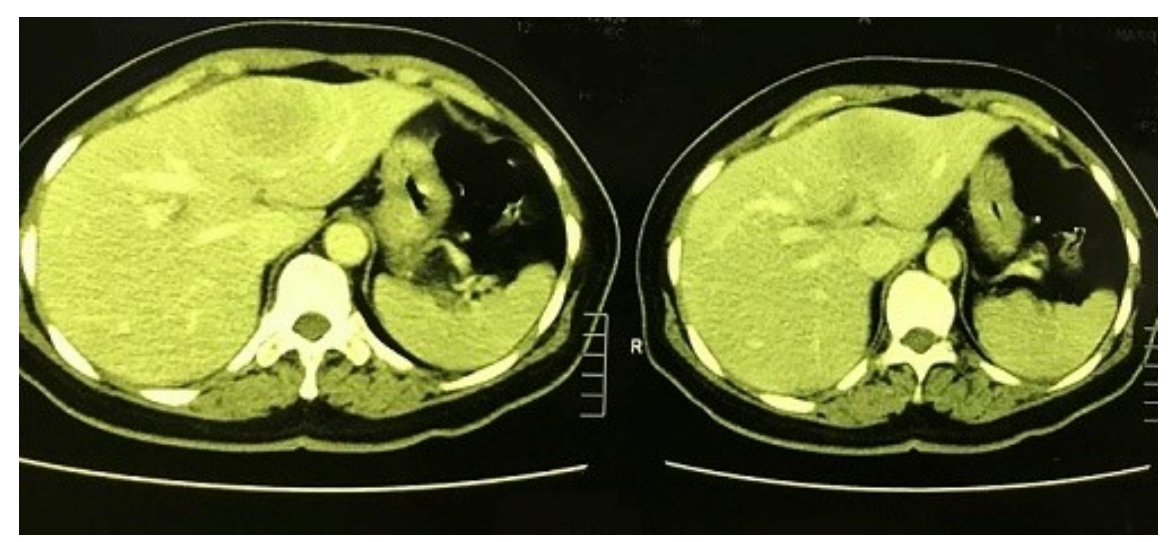



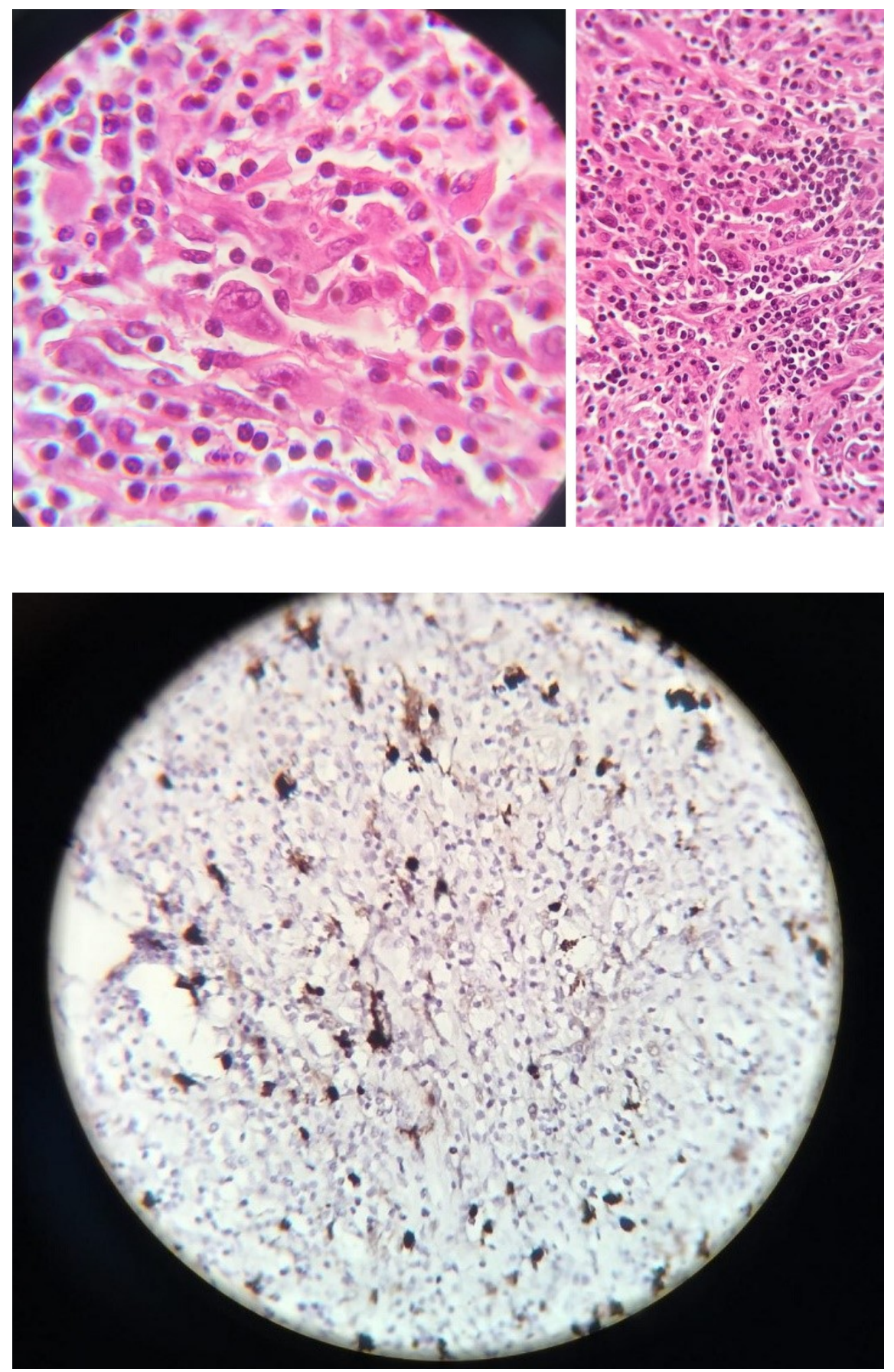